\title{
Ancestral Arithmetic and Isaacson's Thesis
}

\author{
Peter Smith
}

July 9, 2007

\section{Isaacson's Thesis stated}

First-order Peano Arithmetic (PA) is incomplete. So the question naturally arises: what kinds of sentences belonging to PA's language $L_{A}$ can we actually establish to be true even though they are unprovable in PA?

There are two familiar classes of cases. First, there are sentences like the canonical Gödel sentence for PA. Second, there are sentences like the arithmetization of Goodstein's Theorem.

In the first sort of case, we can come to appreciate the truth of the Gödelian undecidable sentences by reflecting on PA's consistency or by coming to accept the instances of the $\Pi_{1}$ reflection schema for PA. And those routes involve deploying ideas beyond those involved in accepting PA as true. To reason to the truth of the Gödel sentence, we need not just to be able to do basic arithmetic, but to be able to reflect on our practice.

In the second sort of case, we come to appreciate the truth of the sentences which are undecidable in PA by deploying transfinite induction or other infinitary ideas. So the reasoning again involves ideas which go beyond what's involved in grasping basic arithmetic.

Thinking about these sorts of cases suggests a plausible general conjecture. Given the arguments of Daniel Isaacson $(1987,1992)$, let's call it

Isaacson's Thesis If we are to give a proof for any true sentence of $L_{A}$ which is independent of PA, then we will need to appeal to ideas that go beyond those that are required in understanding PA.

Isaacson himself famously suggests that the truths of arithmetic that are independent of PA contain 'hidden higher-order concepts'. That way of putting things, however, seems misguided. But be that as it may: it certainly helps to focus discussion if we separate out the core Thesis from Isaacson's further remarks about it.

Is the core Thesis true? In this paper, I'll argue that its fate turns at least in part on the provability or otherwise of a certain technical result. I define a theory I'll call ancestral arithmetic, and will argue that the conceptual resources needed to grasp PA in fact give us a grasp of ancestral arithmetic too. Is this formalized ancestral arithmetic conservative over PA for sentences in the language $L_{A}$ ? If it is, then - so I'll argue that gives some positive support to Isaacson's Thesis. If it isn't, then Isaacson's Thesis certainly fails: there will be truths which are independent of PA but which still can be proved without going beyond what is given us in our understanding of PA.

\section{Second-order arithmetics}

Presented with Isaacson's Thesis cold, you might well think that it faces an immediate and obvious challenge. For you might be struck by Georg Kreisel's often-quoted remark: 
A moment's reflection shows that the evidence of the first-order [induction] schema derives from the second-order [axiom]. (Kreisel, 1972, p. 148)

And if second-order ideas are indeed already involved in our informal understanding of induction, then that suggests an obvious way of going beyond first-order PA while still keeping within the confines of what we already need to understand in accepting PA namely, go second-order.

Now, Isaacson himself says something by way of response to this sort of thought. But let me very quickly put a few points in my own way, which will enable me to mention a theorem - one which is familiar now but wasn't in 1987 - which nicely supports his Thesis.

This much is surely intuitively plausible: we are prepared to give a blanket endorsement to all the instances of the first-order induction schema because we accept the intuitive thought that if zero has some property, and if that property is passed down from one number to the next, then all numbers have that property, whatever property that is. But now ask: what properties are we implicitly quantifying over here?

Well, there is no obvious reason to suppose that our intuitive thought aims to generalize over more than some 'natural' class of arithmetical properties (for example, those expressible by open wffs of $L_{A}$ ). In particular, there is no reason at all to suppose that our intuitive thought aims to use the sophisticated infinitary conception of properties whose extensions can be quite arbitrary subsets of the numbers.

The more cautious mathematicians are alert to exactly this point. For example, Saunders Mac Lane (1986, p. 44) quite explicitly first presents the second-order principle of induction as an induction over the natural arithmetical properties that can be identified by first-order formulae. And he then distinguishes this intuitive 'induction over properties' (as he calls it) from the stronger 'induction over [arbitrary] sets'.

So grant Kreisel his thought that we stand prepared to accept first-order arithmetic with all the instances of its induction schema because we already find ourselves in effect committed to a second-order theory with a second-order induction axiom. That doesn't settle whether the intuitively compelling second-order theory should involve, in Mac Lane's terminology, induction over properties or induction over sets.

Now, the second option, with its infinitary notion of quantification over arbitrary sets of numbers, surely does go far beyond what we are essentially committed to in our grasp of elementary arithmetic - it isn't for nothing that full second-order arithmetic is also known as 'analysis'. But a second-order theory which quantifies only over those arithmetical properties which we can already express in $L_{A}$ arguably is available to someone who grasps arithmetic plus some logical ideas. So here we have intimations of one natural sort of theory of arithmetic which in a sense goes beyond first-order PA while not calling on more sophisticated mathematical concepts. But however exactly we spell that out, such a theory cannot be stronger than the standard second-order arithmetic $\mathrm{ACA}_{0}$ which has a comprehension principle which in effect restricts the domain of the second-order quantifiers to those properties expressible in $L_{A}$. And now here comes the promised theorem: it is now well known that $A C A_{0}$ is conservative over $P A$. The second-order theory does have some very interesting properties of course: for a start, it exhibits massive speed-up over PA (there are PA theorems which have vastly shorter proofs in $\mathrm{ACA}_{0}$ ). But it doesn't prove more $L_{A}$ truths than PA.

In sum, if we go second-order but in a way that deploys ideas that arguably don't go beyond those available in elementary arithmetic plus logic, then we don't get any new truths of $L_{A}$ over and above those which are already available in PA. Which is an observation thoroughly in harmony with Isaacson's Thesis. 


\section{Induction and the ancestral}

So far, so good. But now let's just dig a bit deeper. Even if we restrict the second-order induction axiom to run over kosher arithmetical properties that can be expressed in $L_{A}$, why should we accept it to be true? Why should we stand prepared to accept all the instances of the first-order induction schema?

We've already given the answer. To repeat: if zero has some property, and if that property is passed down from one number to the next, then all numbers have that property. Now note, however, the crucial assumption here: the successors of zero are the only natural numbers. This assumption evidently underlies our acceptance of induction. And, I'd argue, it is an idea which has to be available to anyone who fully grasps PA. For such a grasp involves being able to handle quantifications over the numbers. And understanding quantifications over the numbers involves understanding that the numbers are zero, the next number, the one after that, and so on, without limit - and understanding too that those are the only numbers. Which in effect is to grasp the thought is that every number stands in the ancestral of the successor relation to zero.

And indeed, grasp of the idea of an ancestral - or what comes to much the same, a grasp of the idea of transitive closure under iterable operations - is needed at another place in a full understanding of PA. To appreciate the import of the induction schema, we also need to understand what counts as an open wff of $L_{A}$ that can be plugged into the schema. And to understand what counts as a wff of $L_{A}$ we need to understand the idea of starting from a class of atoms and then applying connectives or prefixing quantifiers, and then doing it again, and doing it again, and so on without limit.

Now, what does it take to grasp the operation of forming the ancestral? Well, we all know that the operation is not first-order definable. Hence, in particular the property of being zero-or-one-of-its-successors, something grasped by someone who understands interpreted PA, is not itself definable in first-order PA. Equally, we all know that the ancestral is definable in a second-order framework (using full quantification over arbitrary subsets of the domain). Thus something has the property of being zero-orone-of-its-successors if it belongs to every set of numbers which contains zero, and if it contains $n$ contains $n^{\prime}$.

Was it wrong, then, so rapidly to dismiss the idea that a grasp of full second-order quantification over arbitrary sets of numbers is built into our understanding of elementary arithmetic? Well, the fact that the notion of the ancestral can be defined in secondorder terms doesn't mean it is an essentially higher-order notion. Here's a comparison. We can define identity in second-order terms by putting $x=y={ }_{\operatorname{def}} \forall X(X x \equiv X y)$. But that certainly doesn't show that understanding identity presupposes an understanding quantification over arbitrary subsets of the domain. It can't show that, because understanding the idea of arbitrary sets of objects of some kind already requires an understanding of what makes for an object of that kind, and that in turn involves an understanding of what makes candidates the same or different objects, i.e. it already involves an understanding of identity.

Similarly for the notion of the ancestral. The child learns that her parents have parents, and that they have parents too. And in sepia tones, her great-grandparents have parents in their turn. And she learns that other children have parents and grandparents too. The penny drops: she realizes that in each case you can keep on going. The child gets the idea of an ancestor, i.e. the idea of being someone who turns up eventually as you trace people back through their parents. And is the idea of the ancestral essentially higher order? Well, it would seem flatly implausible to say that because we can define the idea of an ancestor from the idea of a parent in second-order 
terms, and we can't do it first-order terms - the child who so easily grasps the concept ancestor must already be in cognitive command of the idea of arbitrary sets of people.

Similarly, the child learns to count and do arithmetic. The hundreds are followed by the thousands, and the tens of thousands, and the hundreds of thousands and the millions. Again the penny drops: you can keep on going. And she gets the idea of being a natural number, i.e. of turning up eventually as you keep on moving to the next number. Again, it seems badly over-shooting to say that to get this far, she has to be in cognitive command of the idea of arbitrary sets of numbers.

In sum, the claim is that the child who moves from a grasp of a relation to a grasp of the ancestral of that relation need not thereby manifest an understanding of second-order quantification interpreted as quantification over arbitrary sets. It seems, rather, that she has attained a distinct conceptual level here, something whose grasp requires going beyond a grasp of the fundamental logical constructions regimented in first-order logic, but which doesn't takes as far as an understanding of full second-order quantification.

We don't want to be multiplying conceptual primitives unnecessarily. But, if what we've been saying is right, the concept of the ancestral of a relation doesn't seem at all a bad candidate for being a fundamental logical idea. It is something that can't be explained in simpler terms, but which you can grasp without getting your head round more sophisticated ideas like the classical conception of quantification over arbitrary subsets of a domain. That's not a new thought! - consider Weyl, for example. And for the rest of this paper, let's run with this thought: what happens if we try to build the ancestral operator into a formal theory of arithmetic which is otherwise basically first order?

You can immediately see that raising this question suggests a potential worry for Isaacson's Thesis. For if fully understanding PA involves getting hold of the idea of the ancestral of the successor relation, then an 'ancestral arithmetic' which does have a built-in ancestral operator won't take us beyond what is already within the grasp of someone who really understands PA. So it will matter crucially whether such a theory is or isn't conservative over plain PA. If it isn't then the Thesis is in trouble, for we'll then have a clear counterexample to the claim that proving sentences of $L_{A}$ which are undecided by PA must involve ideas that go beyond those which are constitutive of our understanding of basic arithmetic.

\section{Ancestral arithmetic}

Let's start thinking, then, how we could extend a first-order language with an ancestralforming operator.

To begin, we need to expand our first-order logical vocabulary with an operator we'll symbolize it with a star - which attaches to two-place expressions $\varphi(x, y): \varphi^{*}(x, y)$ is to be interpreted as expressing the ancestral of the original relation expressed by $\varphi(x, y)$. We'll be forgivably careless about the syntactic details here.

Suppose that we augment the language $L_{A}$ with such an operator. Abbreviate $y=x^{\prime}$ by $S x y$. And first consider then what happens when you take the familiar axioms of PA but add in the new axiom

$$
\text { S. } \forall x\left(x=0 \vee S^{*} 0 x\right)
$$

which says - as we want - that every number is zero or one of its successors.

Any interpretation of this expanded set of axioms which respects the now-to-bekept-fixed logical meaning of the ancestral-forming operator evidently must be slim, that is to say, have just the 'zero' and its 'successors' in the domain. But, by a standard 
argument, the slim models of the remaining axioms of PA are all isomorphic. So our augmented theory is categorical.

Let's define, then, the appropriate semantic entailment relation $\Gamma \vDash * \varphi$, which obtains if every interpretation which makes all of $\Gamma$ true makes $\varphi$ true - where we are now generalizing just over interpretations which give the star operator its intended meaning (and otherwise treats the logical vocabulary standardly). Then, because of categoricity, our expanded axioms semantically entail any true sentence of the expanded language. So in particular, if $\varphi$ is a true sentence of the unexpanded language $L_{A}$, then $\mathrm{PA}+\mathrm{S} \vDash * \varphi$.

There can't, however, be a complete axiomatization of this 'ancestral arithmetic'. That follows by a simple compactness argument (for $\vDash^{*}$ is evidently non-compact). Or we can argue as follows. Take any formally axiomatized theory which is sound for $\vDash^{*}$ and which includes PA. The incompleteness theorem then applies (assuming consistency), so there will be an unprovable-yet-true $L_{A}$ sentence $G^{*}$ for this theory. But since the $L_{A}$ truths are all semantically entailed by ancestral arithmetic, this means that there is an unprovable-yet-semantically-entailed sentence, so the deductive system can't be complete.

So far, this ought to familiar from the last chapter of Stewart Shapiro's classic book on second-order logic (1991). But now note that the unaxiomatizability of $\vDash^{*}$ of course doesn't rule out partial axiomatizations of ancestral arithmetic. Shapiro doesn't explore the options here: but R. M. Martin and John Myhill did, in a series of articles in JSL going back to 1943 (Martin, 1943, 1949), (Myhill, 1952).

Suppose we write $H(\psi, \varphi)$ as short for $\forall x \forall y((\psi(x) \wedge \varphi(x, y)) \rightarrow \psi(y))$ - which says that the property expressed by $\psi$ is hereditary with respect to the relation $\varphi$. Then Myhill's proposed axioms for the star operator are tantamount to the following schematic rules:

From $\varphi(a, b)$ infer $\varphi^{*}(a, b)$.

From $\varphi^{*}(a, b), \varphi(b, c)$ infer $\varphi^{*}(a, c)$.

From $H(\psi, \varphi)$, infer $H\left(\psi, \varphi^{*}\right)$.

These first two rules serve like 'introduction' rules for the star operator; and the third rule is easily seen to be equivalent to the following 'elimination' rule:

From $\varphi^{*}(a, b)$ infer $\{\psi(a) \wedge H(\psi, \varphi)\} \rightarrow \psi(b)$.

(Of course, when I call those 'introduction' and 'elimination' rules, I don't mean to imply that they are rules that suffice to fix the content of the star operator - they can't, given that the rules aren't complete.)

Our 'elimination' rule is evidently a kind of generalized inductive principle which says that given that $b$ is a $\varphi$ descendant of $a$ then if $a$ has some property which is passed down from one thing to another if they are $\varphi$ related, then $b$ has that property too. And it is easy to see that - in the presence of these rules and the axiom $\mathrm{S}$ - our generalized induction principle entails all instances of the ordinary first-order induction schema.

So let's now consider the formal system PA* which extends Robinson Arithmetic $\mathrm{Q}$ by adding our new axiom (S) plus Myhill's rules for handling the ancestral operator (and so also extends PA). The obvious next question is: what is the deductive power of this system with respect to sentences of $L_{A}$ ? Is this another case like the canonical partial axiomatization of full second-order arithmetic, $\mathrm{PA}_{2}$ (a.k.a. $\mathrm{Z}_{2}$ ), where we also only have a partial axiomatization of the relevant semantic consequence relation, though axiomatized $\mathrm{PA}_{2}$ can prove more $L_{A}$ sentences than PA (e.g. it can prove $C o n_{\mathrm{PA}}$ ). Or is $\mathrm{PA}^{*}$ another extension of $\mathrm{PA}$ like $\mathrm{ACA}_{0}$, which is conservative over $\mathrm{PA}$ ? 
If the first case holds, then - as I said - we'd surely have a refutation of Isaacson's Thesis. For PA* is arguably well within the conceptual reach of someone who has fully understood the inductive schema of PA; and so, if we could use it to prove new sentences of basic arithmetic not provable in PA, Isaacson's Thesis would fail.

\section{$5 \quad \mathrm{PA}^{*}$ is conservative over $\mathrm{PA}$}

In fact, things do fall out Isaacson's way. Here's a theorem (which I haven't found in the literature, but is straightforward):

$$
P A^{*} \text { is conservative over } P A \text {. }
$$

In other words, for any $L_{A}$ sentence $\psi$ such that $\mathrm{PA}^{*} \vdash \psi$, it is already the case that $P A \vdash \psi$.

Proof sketch Recall that we can express facts about sequences of numbers in PA by using a $\beta$-function where for any finite sequence $k_{0}, k_{1}, \ldots, k_{x}$ there is some $c$ such that for all $u \leq x, \beta(c, u)=k_{u}$. So suppose $R$ is some relation. Then its ancestral $R^{*}$ is such that

A. $R^{*} a b$ is true just so long as, for some $x$, there is a sequence of numbers $k_{0}, k_{1}, \ldots, k_{x}$ such that: $k_{0}=a$, and if $u<x$ then $R k_{u} k_{S u}$, and $k_{x}=b$.

Using a $\beta$-function, that means

B. $R^{*} a b$ is true iff for some $x$, there is a $c$ such that: $\beta(c, 0)=a$, and if $u<x$ then $R(\beta(c, u), \beta(c, S u))$, and $\beta(c, x)=b$.

So consider the following definition:

$$
\begin{aligned}
& \text { C. } \varphi^{* *}(a, b)={ }_{\text {def }} \exists x \exists c\{B(c, 0, a) \wedge \\
&(\forall u<x) \exists v \exists w\{(B(c, u, v) \wedge B(c, S u, w)) \wedge \varphi(v, w)\} \wedge \\
& \\
&B(c, x, b)\}
\end{aligned}
$$

where $\varphi$ expresses some relation $R$, and $B$ is a formal three-place wff of $L_{A}$ which captures a $\beta$-function. Then $\varphi^{* *}$ expresses the ancestral $R^{*}$ of the relation $R$ expressed by $\varphi$.

It is now easy to check that the Myhill inference rules for starred wffs apply equally to our defined double-starred construct $\varphi^{* *}$ in PA (that's because the moves are then evidently valid semantic entailments within PA, and the theory's deductive system is complete), and the double-starred analogue of axiom (S) is also a theorem of PA. So corresponding to any proof involving starred wffs in $\mathrm{PA}^{*}$ there is an exactly parallel proof in plain PA involving double-starred wffs. Hence, in particular, any proof of a pure (i.e. star-less) $L_{A}$ wff in $\mathrm{PA}^{*}$ has a parallel proof in plain PA. QED.

\section{Other ancestral arithmetics?}

But is PA* (or some variant of it, which can similarly be proved to be conservative over PA) as far as we can get solely by recourse to ideas already involved in the understanding of PA? Well, the literature on ancestral arithmetics is surprisingly thin. To be sure, there is an extensive treatment of logics with a transitive closure operator in the literature on finite model theory; but that is all model-theoretic, and doesn't seem to discuss deductive systems. There's a paper by Arnon Avron (2003) which in effect rediscovers Myhill's rules, and then puts it down as an open question what other 
rules for the star operator should be added "in order to make the system "complete" in some reasonable sense'. And there is a passing discussion in Richard Heck (2007), but Heck's interest is in stronger systems which draw on essentially richer conceptual resources. So I don't know of other essentially stronger competitors to PA* for being formally axiomatized regimentations of what is available to us through our grasp of the ancestral. Given that the conservativeness proof using the $\beta$-function trick generalizes in obvious ways, I conjecture that there aren't any.

\section{Conclusion}

Let's summarize. Our everyday understanding of basic arithmetic pins down a unique structure for the natural numbers, at least up to isomorphism. Hence our grasp of basic arithmetic involves more than is captured by first-order PA. But what more? It seems enough that we have the idea that the natural numbers are zero and its successors and nothing else. And getting our head round this idea, we suggested, involves the general idea of the ancestral: the numbers are what stand in the ancestral of the successor relation to zero.

Now, the ancestral of a relation can be defined in second-order terms, but it seems overkill to suppose that our understanding of ordinary school arithmetic is essentially second-order. Why not treat the operation that takes a relation to its ancestral to be a (relatively unmysterious, even though not purely first-order) logical primitive? If we do, we can construct a formalized theory PA* which naturally extends PA in a way that arguably still reflects our everyday understanding of arithmetic.

Since $\mathrm{PA}^{*}$ is still properly axiomatized, however, we know that it will be incomplete (assuming it is consistent). But we might have suspected that it would at least have proved more arithmetic truths than PA: but not so. PA* is deductively conservative over PA for $L_{A}$ sentences: so we can't in fact use this expanded theory to deduce new truths of basic arithmetic that are left unsettled by PA.

Hence, to put it the other way around, it seems that if we are to come up with proofs of truths unsettled by PA, then we'll have to deploy premisses and/or logical apparatus that go beyond $\mathrm{PA}^{*}$. Which (at least if the conjecture at the end of the last section is correct) implies that we'll need to invoke ideas which go beyond those essential to our ordinary understanding of basic arithmetic. Why? Because - apart from a grasp of the recursive definitions of addition and multiplication - the idea that all the numbers can be reached from zero by repeatedly adding one, i.e. the idea that all numbers are related to zero by the ancestral of the successor relation, does seem to be at the limit of what is necessary to the grasp of the purely arithmetic concepts which underlie PA.

And if that's right, Isaacson's Thesis looks secure. ${ }^{1}$

\section{References}

Avron, A., 2003. Transitive closure and the mechanization of mathematics. In F. Kamareddine (ed.), Thirty Five Years of Automating Mathematics, pp. 149-171. Dordrecht: Kluwer Academic Publishers.

Heck, R., 2007. The logic of Frege's Theorem. Forthcoming in a Festschrift for Crispin Wright.

\footnotetext{
${ }^{1}$ Particular thanks to Andreas Blass, Ben Colburn, Dan Isaacson and Aatu Koskensilta.
} 
Isaacson, D., 1987. Arithmetical truth and hidden higher-order concepts. In The Paris Logic Group (ed.), Logic Colloquium '85. Amsterdam: North-Holland.

Isaacson, D., 1992. Some considerations on arithmetical truth and the $\omega$-rule. In M. Detlefsen (ed.), Proof, logic and formalization, pp. 94-138. London: Routledge.

Kreisel, G., 1972. Informal rigour and completeness proofs. In I. Lakatos (ed.), Problems in the Philosophy of Mathematics. Amsterdam: North-Holland.

Mac Lane, S., 1986. Mathematics: Form and Function. New York: Springer-Verlag.

Martin, R. M., 1943. A homogeneous system for formal logic. Journal of Symbolic Logic, 8: 1-23.

Martin, R. M., 1949. A note on nominalism and recursive functions. Journal of Symbolic Logic, 14: 27-31.

Myhill, J., 1952. A derivation on number theory from ancestral theory. Journal of Symbolic Logic, 17: 192-197.

Shapiro, S., 1991. Foundations without Foundationalism: A Case for Second-Order Logic. Oxford Logic Guides 17. Oxford: Clarendon Press. 